\title{
POINTWISE A POSTERIORI ERROR ESTIMATES FOR MONOTONE SEMI-LINEAR EQUATIONS
}

\author{
RICARDO H. NOCHETTO, ALFRED SCHMIDT, KUNIBERT G. SIEBERT, \\ AND ANDREAS VEESER
}

\begin{abstract}
We derive upper and lower a posteriori estimates for the maximum norm error in finite element solutions of monotone semi-linear equations. The estimates hold for Lagrange elements of any fixed order, non-smooth nonlinearities, and take numerical integration into account. The proof hinges on constructing continuous barrier functions by correcting the discrete solution appropriately, and then applying the continuous maximum principle; no geometric mesh constraints are thus required. Numerical experiments illustrate reliability and efficiency properties of the corresponding estimators and investigate the performance of the resulting adaptive algorithms in terms of the polynomial order and quadrature.
\end{abstract}

\section{INTRODUCTION}

Adaptive finite elements methods (AFEM) are a popular and efficient method for the approximation of solutions to partial differential equations (PDE). A crucial theoretical step in designing these methods are a posteriori error estimates that relate the error to quantities that are computable in terms of the discrete solution and data. For an overview on these methods, techniques, and their development, we refer to the books $[2,26]$.

Most a posteriori error estimates have been derived for the energy norm error. In contrast, the pointwise error has been investigated much less and, up to now, only for linear finite elements: $[10,17]$ analyze the linear elliptic problem, while $[19,20]$ treat the elliptic obstacle problem.

In this article we consider the Dirichlet problem for a monotone semi-linear PDE,

$$
-\Delta u+f(\cdot, u)=0 \quad \text { in } \Omega, \quad u=g \quad \text { on } \partial \Omega,
$$

in a polyhedral domain $\Omega \subset \mathbb{R}^{d}$ with $d \geq 2$, and approximate its solution with continuous finite elements of any fixed polynomial degree; we thus study the socalled $h$-AFEM. Instead of the Laplacian $\Delta$, we could examine more general elliptic operators with variable coefficients, but the analysis would inevitably get much more involved, which we want to avoid here. Denoting the finite element solution by $u_{h}$, our main result (see Theorem 4.2 ) reads

$$
\left\|u-u_{h}\right\|_{\infty ; \Omega} \leq \eta_{\infty}+\eta_{d}+\eta_{d / 2} .
$$

The contribution $\eta_{\infty}$ estimates the residual in an appropriate dual norm and the approximation of the boundary values, while $\eta_{d}+\eta_{d / 2}$ estimates the error due to

Date: July 31, 2006.

1991 Mathematics Subject Classification. 65N15, 65N30, 35J85.

Key words and phrases. semi-linear equations, a posteriori error estimate, residual, maximum norm, maximum principle, numerical integration, Lagrange elements. 
numerical integration. The proof of (1.2) hinges on the construction of barrier functions for (1.1). They are obtained by suitably altering the discrete solution $u_{h}$ via the Riesz representation $w \in \stackrel{\circ}{H}^{1}(\Omega)$ of the residual, namely $u_{h} \pm w$, plus additional constant corrections (vertical shifts). This idea has already been used by Nochetto, Siebert, and Veeser for the elliptic obstacle problem [19, 20], but the analysis here is simpler and thus more transparent. Complementing local lower bounds are also established (see Theorem 4.3). Several comments are in order:

- For the linear case, where $f$ does not depend on its second argument, the aforementioned estimates generalize $[10,17]$ in two aspects: the polynomial degree is not restricted to one and numerical integration is taken into account. They are both novel features in maximum norm error analysis.

- The use of numerical integration for (1.1) corresponds to the discretization of the constraint in the obstacle problem, where the nonlinearity $f$ is a maximal monotone graph. The treatment of quadrature here and discretization of the constraint in $[19,20]$ are different. We propose a novel multidegree splitting which evaluates a posteriori the effect of quadrature and does not require smoothness of $f(x, \cdot)$ beyond continuity (see $\S 3.2)$.

- In (1.2) the contribution $\eta_{p}, p=d / 2, d, \infty$, is the $\ell_{p}$-norm of the corresponding local indicators. These contributions thus "accumulate" in different ways. An estimator with such a property was also used in [19] and handled with an appropriate two-step marking strategy. Here we use a similar strategy and investigate its performance in Remark 4.4 and $\S 5$.

- Since we use the continuous maximum principle to deal with barrier functions, our current results are neither restricted to polynomial degree one nor impose any geometric mesh constraints such as weak acuteness.

We study the Riesz representation $w$ of the residual in $\S 3$, whereas we construct barrier functions and prove error estimates in $\S 4$. We conclude in $\S 5$ with several numerical experiments which explore reliability, efficiency, and performance of AFEM for different polynomial degrees, quadrature, and interesting model problems; the implementation has been realized within the toolbox ALBERTA [24, 25].

\section{The Continuous Problem and its Discretization}

Let $\Omega$ be a bounded, polyhedral, not necessarily convex domain in $\mathbb{R}^{d}$ with $d \geq 2$. The nonlinearity $f: \bar{\Omega} \times \mathbb{R} \rightarrow \mathbb{R}$ is assumed to be continuous in $\bar{\Omega} \times \mathbb{R}$ and non-decreasing in the second argument; however, we could also treat a piecewise continuous $f$ in the first argument, with respect to the underlying mesh. The Dirichlet boundary datum $g$ satisfies $g \in H^{1}(\Omega) \cap C^{0, \alpha}(\bar{\Omega})$ with $0<\alpha \leq 1$.

Let $u$ be a weak solution of (1.1). More precisely, if we write $\langle\varphi, \psi\rangle$ for $\int_{\Omega} \varphi \psi$, then $u$ satisfies

$$
u \in g+\stackrel{\circ}{H}^{1}(\Omega): \quad\langle\nabla u, \nabla v\rangle+\langle f(\cdot, u), v\rangle=0 \quad \text { for all } v \in \stackrel{\circ}{H}^{1}(\Omega) .
$$

It is known that such $u$ exists, is unique, and is bounded [7, Lemma 16], [11, Section 9.3], [15, Section III.3]. Since $x \mapsto f(x, u(x))$ is thus a bounded function, regularity theory for linear elliptic PDE ensures that $u$ is Hölder continuous in $\bar{\Omega}$ $[11,12,13,15]$.

Therefore, it makes sense to approximate $u$ in $L_{\infty}(\Omega)$ with continuous finite elements. We shall use elements of any fixed order and numerical integration for the

nonlinearity $f$. Given a conforming and shape-regular triangulation $\mathcal{T}_{h}$ of $\bar{\Omega}$, made 
of closed elements $T$, let $\mathbb{V}_{h}$ indicate the space of continuous piecewise polynomial finite element functions of degree $\ell \geq 1$. We set $\stackrel{\bullet}{V}_{h}:=\mathbb{V}_{h} \cap \stackrel{\leftrightarrow}{H}^{1}(\Omega)$ and $g_{h}:=I_{h} g$, where $I_{h}$ is the Lagrange interpolation operator onto $\mathbb{V}_{h}$.

For $\varphi, \psi \in C^{0}(\bar{\Omega})$, we define

$$
\langle\varphi, \psi\rangle_{h}:=\sum_{T \in \mathcal{T}_{h}} Q_{T}(\varphi \psi)
$$

where $Q_{T}$ is a quadrature formula for the integral over $T \in \mathcal{T}_{h}$ that is induced by a fixed quadrature formula $\hat{Q}$ on a reference element $\hat{T}$. We suppose that $\hat{Q}$ has positive weights, is exact for polynomials of degree $q$ with

$$
q \geq \max \{2 \ell-2,1\}
$$

and that all quadrature points are contained in $\hat{T} ; q \geq 1$ is the order of $\hat{Q}$. Restriction (2.3) is consistent with the a priori analysis [9, Section 4.1], and is crucial to obtain optimal convergence rates for uniform refinement which can be a particular case of adaptive refinement.

The discrete problem then reads as follows:

$$
u_{h} \in g_{h}+\stackrel{\circ}{V}_{h}: \quad\left\langle\nabla u_{h}, \nabla v_{h}\right\rangle+\left\langle f\left(\cdot, u_{h}\right), v_{h}\right\rangle_{h}=0 \quad \text { for all } v_{h} \in \stackrel{\circ}{V}_{h} .
$$

Thanks to the positivity of the weights in the quadrature formulae, the discrete nonlinearity is monotone and thus Problem (2.4) admits a unique solution; see [21].

\section{Estimation of Residual and its Riesz Representation}

Key ingredients of our a posteriori error analysis are the residual $\mathcal{R}_{h} \in H^{-1}(\Omega)$,

$$
\begin{aligned}
\left\langle\mathcal{R}_{h}, \varphi\right\rangle & =-\left\langle\nabla u_{h}, \nabla \varphi\right\rangle-\left\langle f\left(\cdot, u_{h}\right), \varphi\right\rangle \\
& =\left\langle\nabla\left(u-u_{h}\right), \nabla \varphi\right\rangle+\left\langle f(\cdot, u)-f\left(\cdot, u_{h}\right), \varphi\right\rangle
\end{aligned}
$$

for all $\varphi \in \stackrel{\circ}{H}^{1}(\Omega)$, and its Riesz representation $w \in \stackrel{\circ}{H}^{1}(\Omega)$ satisfying

$$
\langle\nabla w, \nabla \varphi\rangle=\left\langle\mathcal{R}_{h}, \varphi\right\rangle \quad \text { for all } \varphi \in \stackrel{\circ}{H}^{1}(\Omega) .
$$

In this section, we first establish a posteriori estimates of $\mathcal{R}_{h}$ in negative norms which are then used to derive pointwise estimates for $w$. Correcting the discrete solution $u_{h}$ with $w$ yields barriers for the true solution $u$. These barriers combined with the pointwise control of $w$ by estimates of $\mathcal{R}_{h}$ finally lead to a posteriori control of the pointwise error $\left\|u-u_{h}\right\|_{\infty ; \Omega}$; see $\S 4.1$ and $\S 4.2$.

3.1. Estimating the residual. We start by introducing some notation. Let $\Gamma_{h}$ be the union of (closed) inter-element sides (edges in $2 \mathrm{~d}$ and faces in $3 \mathrm{~d}$, respectively) of $\mathcal{T}_{h}$ and $\llbracket \partial_{n} u_{h} \rrbracket$ be the jumps of the normal derivatives of $u_{h}$ across inter-element sides. More precisely, given a common side $\gamma=T^{+} \cap T^{-} \subset \Gamma_{h}$, we have on $\gamma$

$$
\llbracket \partial_{n} u_{h} \rrbracket=\left(\nabla u_{h \mid T^{+}}-\nabla u_{h \mid T^{-}}\right) \cdot n,
$$

where $n$ is the normal of $\gamma$ that points from $T^{-}$to $T^{+}$.

Let $h_{T}$ be the diameter of an element $T$ and denote by $h \in L_{\infty}(\Omega)$ the piecewise constant function with $h_{\mid T}=h_{T}, T \in \mathcal{T}_{h}$; recall that elements $T \in \mathcal{T}_{h}$ are closed. In the following analysis the symbol ' $\preccurlyeq$ ' stands for ' $\leq C$ ', where the generic constant $C$ does not depend on $h$ but may depend on

- the shape-regularity of the partition $\mathcal{T}_{h}$,

- the quadrature formula defined on the reference element, 
- the domain $\Omega$ and its dimension $d$.

By $\Pi_{h}$ we denote the Clement interpolation operator into the space of continuous finite element functions that are piecewise affine over $\mathcal{T}_{h}$ and have homogeneous boundary values. The following properties are valid for all $1 \leq p \leq \infty, T \in \mathcal{T}_{h}$

$$
\begin{aligned}
& \left\|\Pi_{h} \varphi\right\|_{p ; T} \preccurlyeq\|\varphi\|_{p ; \mathcal{U}_{h}(T)} \quad \varphi \in L_{p}(\Omega), \\
& \left\|\nabla \Pi_{h} \varphi\right\|_{p ; T} \preccurlyeq\|\nabla \varphi\|_{p ; \mathcal{U}_{h}(T)}, \quad \varphi \in \stackrel{\circ}{W}_{p}^{1}(\Omega), \\
& \left\|\varphi-\Pi_{h} \varphi\right\|_{p ; T} \preccurlyeq h_{T}\|\nabla \varphi\|_{p ; \mathcal{U}_{h}(T)} \quad \varphi \in \stackrel{\circ}{W}_{p}^{1}(\Omega), \\
& \left\|\varphi-\Pi_{h} \varphi\right\|_{1 ; T} \preccurlyeq h_{T}^{2}\left\|D^{2} \varphi\right\|_{1 ; \mathcal{U}_{h}(T)} \quad \varphi \in W_{1}^{2}(\Omega) \cap \stackrel{\circ}{H}^{1}(\Omega) .
\end{aligned}
$$

Hereafter, $\mathcal{U}_{h}(T)$ denotes the set of all triangles $T^{\prime} \in \mathcal{T}_{h}$ that have a non-empty intersection with $T$.

We now start by estimating the residual $\mathcal{R}_{h}$. In view of the definition (2.4) of $u_{h}$, we may write

$$
\left\langle\mathcal{R}_{h}, \varphi\right\rangle=\left\langle\mathcal{R}_{h}, \varphi-\Pi_{h} \varphi\right\rangle+\left\langle f\left(\cdot, u_{h}\right), \Pi_{h} \varphi\right\rangle_{h}-\left\langle f\left(\cdot, u_{h}\right), \Pi_{h} \varphi\right\rangle
$$

for all $\varphi \in \stackrel{\circ}{H}^{1}(\Omega)$. The estimation of the first term is fairly standard; for convenience of the reader, we give the main steps. Piecewise integration by parts yields

$$
\left\langle\mathcal{R}_{h}, \varphi-\Pi_{h} \varphi\right\rangle=\int_{\Gamma_{h}} \llbracket \partial_{n} u_{h} \rrbracket\left(\varphi-\Pi_{h} \varphi\right)+\int_{\Omega}\left[\Delta u_{h}-f\left(\cdot, u_{h}\right)\right]\left(\varphi-\Pi_{h} \varphi\right) .
$$

Here, $\Delta u_{h}$ has to be understood element-wise. To simplify notation, we define for any $1 \leq p \leq \infty$ the residual indicator $R_{p}$ over $T \in \mathcal{T}_{h}$ by

$$
R_{p \mid T}:=h_{T}^{-1 / p^{\prime}}|T|^{-1 / p}\left\|\llbracket \partial_{n} u_{h} \rrbracket\right\|_{p ; \partial T \backslash \partial \Omega}+\left|\Delta u_{h}-f\left(\cdot, u_{h}\right)\right|,
$$

where $p^{\prime}=p /(p-1)$ is the dual exponent of $p$ (with the usual conventions for $p=1, \infty)$; the first term is the jump residual and the second one is the interior residual.

Lemma 3.1 (Residual estimates). For any $1 \leq p \leq \infty$, we have

$$
\begin{array}{ll}
\left\langle\mathcal{R}_{h}, \varphi-\Pi_{h} \varphi\right\rangle \preccurlyeq\left\|h^{2} R_{\infty}\right\|_{\infty ; \Omega}\left\|D^{2} \varphi\right\|_{1 ; \Omega}, & \text { for all } \varphi \in W_{1}^{2}(\Omega) \cap \stackrel{\circ}{H}^{1}(\Omega) \\
\left\langle\mathcal{R}_{h}, \varphi-\Pi_{h} \varphi\right\rangle \preccurlyeq\left\|h R_{p}\right\|_{p ; \Omega}\|\nabla \varphi\|_{p^{\prime} ; \Omega} \quad \text { for all } \varphi \in \stackrel{\circ}{W}_{p^{\prime}}^{1}(\Omega) .
\end{array}
$$

Proof. Estimate the right hand side of (3.5) with the help of the stability and approximation properties of the Clement interpolant (3.3) as well as a scaled trace inequality.

3.2. Estimating the quadrature error. Let us turn to the last two terms in (3.4), which are related to the quadrature error. Defining $\mathcal{E}_{h} \in H^{-1}(\Omega)$ by

$$
\left\langle\mathcal{E}_{h}, \varphi\right\rangle=\left\langle f\left(\cdot, u_{h}\right), \Pi_{h} \varphi\right\rangle_{h}-\left\langle f\left(\cdot, u_{h}\right), \Pi_{h} \varphi\right\rangle \quad \text { for all } \varphi \in \stackrel{\circ}{H}^{1}(\Omega),
$$

we have

$$
\left\langle\mathcal{E}_{h}, \varphi\right\rangle=\sum_{T \in \mathcal{T}_{h}}\left[Q_{T}\left(f\left(\cdot, u_{h}\right) \Pi_{h} \varphi\right)-\int_{T} f\left(\cdot, u_{h}\right) \Pi_{h} \varphi\right]=: \sum_{T \in \mathcal{T}_{h}} E_{T}\left(f_{h} \varphi_{h}\right) .
$$

Hereafter, we use the abbreviations $f_{h}:=f\left(\cdot, u_{h}\right), \varphi_{h}:=\Pi_{h} \varphi$, and $E_{T}(\cdot)$ stands for the local quadrature error on $T, T \in \mathcal{T}_{h}$. In view of our assumptions on $Q_{T}$, 
the error $E_{T}$ satisfies

$$
\left|E_{T}(\psi)\right|=\left|Q_{T}(\psi)-\int_{T} \psi\right| \leq 2|T|\|\psi\|_{\infty ; T} \quad \text { for all } \psi \in C^{0}(T)
$$

and

$$
E_{T}(\psi)=0 \quad \text { for all } \psi \in \mathbb{P}_{q}(T) .
$$

In order to proceed further, it is useful to notice the following facts:

- the cancellations (3.10b) in the quadrature error are related to the product $f_{h} \varphi_{h}$ and not only to $f_{h}$ or the discrete test function $\varphi_{h}$;

- in general, $f_{h}=f\left(\cdot, u_{h}\right)$ is not polynomial and thus usually $f_{h} \varphi_{h}$ can not be integrated exactly;

- we do not suppose that the nonlinearity $f$ is differentiable; hence, an application of the Bramble-Hilbert lemma involving derivatives of $f_{h} \varphi_{h}$ and thus of $f$ is not possible.

We overcome these difficulties by a multidegree splitting of the consistency error using different interpolation/projection operators. The idea is based on the method used in [9, Section 4.1] for estimating the quadrature error in the a priori analysis.

Lemma 3.2 (Consistency estimates). Let $q \geq \ell$ be the quadrature order. For $\psi \in$ $C^{0}(\bar{\Omega})$ and $j=q, q-1$ let $I_{h}^{j} \psi$ be any element-wise approximation of $\psi$ satisfying $I_{h}^{j} \psi_{\mid T}$ in $\mathbb{P}_{j}(T), T \in \mathcal{T}_{h}$. For $1 \leq p \leq \infty$, let $|\cdot|_{p}$ be the $\ell_{p}\left(\mathbb{R}^{\# \mathcal{T}_{h}}\right)$ norm and $\varepsilon_{p}^{j}$ the sequence given by

$$
\varepsilon_{p, T}^{j}=|T|^{1 / p}\left\|f\left(\cdot, u_{h}\right)-I_{h}^{j} f\left(\cdot, u_{h}\right)\right\|_{\infty ; T} \quad \text { for all } T \in \mathcal{T}_{h} .
$$

The following estimates for the consistency error hold

$$
\left\langle\mathcal{E}_{h}, \varphi\right\rangle \preccurlyeq\left[\left|\varepsilon_{d / 2}^{q}\right|_{d / 2}+\left|h \varepsilon_{d}^{q-1}\right|_{d}\right]\left\|D^{2} \varphi\right\|_{1 ; \Omega}
$$

for all $\varphi \in W_{1}^{2}(\Omega) \cap \stackrel{\circ}{H}^{1}(\Omega)$ and

$$
\left\langle\mathcal{E}_{h}, \varphi\right\rangle \preccurlyeq\left[\left|h^{-1} \varepsilon_{d / 2}^{q}\right|_{d / 2}+\left|\varepsilon_{d}^{q-1}\right|_{d}\right]\|\nabla \varphi\|_{p^{\prime} ; \Omega}
$$

for all $\varphi \in \stackrel{\circ}{W}_{p^{\prime}}^{1}(\Omega)$ and $1 \leq p \leq \infty$ with $p^{\prime}=p /(p-1)$ the dual exponent of $p$.

Remark 3.3 (Choice of $I_{h}^{j}$ ). The optimal choice for $I_{h}^{j} f_{h_{\mid T}}$ would be the best approximation in $L_{\infty}$ of $f_{h}=f\left(\cdot, u_{h}\right)$ in $\mathbb{P}_{j}(T)$. In general, for $j>0$ this best approximation is not easy to compute. Since $I_{h}^{j}$ can be any approximation operator, we have used the computationally convenient Lagrange interpolation operator into the space of piecewise polynomials of degree $\leq j$ in our implementation. Note that, in view of the stability of Lagrange interpolation in $C^{0}$, this is a quasi-optimal choice.

Proof of Lemma 3.2. In addition to the operators $I_{h}^{j}$ we need the $L_{2}$-projection $P_{h}^{0}$ onto the space of piecewise constant functions over $\mathcal{T}_{h}$, i. e. $P_{h}^{0} \psi_{\mid T}=\frac{1}{|T|} \int_{T} \psi$ for $\psi \in L_{2}(\Omega)$ and $T \in \mathcal{T}_{h}$. As a first step, we decompose the discrete test function $\varphi_{h}$ into

$$
\varphi_{h}=P_{h}^{0} \varphi_{h}+\left[\varphi_{h}-P_{h}^{0} \varphi_{h}\right]
$$


Using representation (3.9) along with (3.10b) - quadrature $Q_{T}$ is exact of degree $q \geq 1$ - and the fact that $\varphi_{h \mid T} \in \mathbb{P}_{1}(T)$ for all $T \in \mathcal{T}_{h}$, this splitting implies

$$
\begin{aligned}
\left\langle\mathcal{E}_{h}, \varphi\right\rangle & =\sum_{T \in \mathcal{T}_{h}} E_{T}\left(f_{h} \varphi_{h}\right)=\sum_{T \in \mathcal{T}_{h}}\left[E_{T}\left(f_{h} P_{h}^{0} \varphi_{h}\right)+E_{T}\left(f_{h}\left[\varphi_{h}-P_{h}^{0} \varphi_{h}\right]\right)\right] \\
& =\sum_{T \in \mathcal{T}_{h}} E_{T}\left(\left[f_{h}-I_{h}^{q} f_{h}\right] P_{h}^{0} \varphi_{h}\right)+\sum_{T \in \mathcal{T}_{h}} E_{T}\left(\left[f_{h}-I_{h}^{q-1} f_{h}\right]\left[\varphi_{h}-P_{h}^{0} \varphi_{h}\right]\right) .
\end{aligned}
$$

We now estimate the sums on the right hand side of (3.13) separately and start with the first one. The stability of quadrature (3.10a) readily implies

$$
E_{T}\left(\left[f_{h}-I_{h}^{q} f_{h}\right] P_{h}^{0} \varphi_{h}\right) \leq 2 h_{T}^{d}\left\|f_{h}-I_{h}^{q} f_{h}\right\|_{\infty ; T}\left\|P_{h}^{0} \varphi_{h}\right\|_{\infty ; T}
$$

for each element $T \in \mathcal{T}_{h}$. Using the stability of $P_{h}^{0}$ in $L_{\infty}(T)$, an inverse estimate, and the local stability property $(3.3 \mathrm{a})$ of $\Pi_{h}$, we obtain for the second factor

$$
\left\|P_{h}^{0} \varphi_{h}\right\|_{\infty ; T} \leq\left\|\varphi_{h}\right\|_{\infty ; T} \preccurlyeq h_{T}^{2-d}\left\|\varphi_{h}\right\|_{d /(d-2) ; T} \preccurlyeq h_{T}^{2-d}\|\varphi\|_{d /(d-2) ; \mathcal{U}_{h}(T)},
$$

using the convention $d /(d-2)=\infty$ for $d=2$. Thus summing over $T \in \mathcal{T}_{h}$, the Hölder inequality in $\mathbb{R}^{\# \mathcal{T}_{h}}$ with $p=d / 2$ and $p^{\prime}=d /(d-2)$ gives

$$
\begin{aligned}
\sum_{T \in \mathcal{T}_{h}} & E_{T}\left(\left[f_{h}-I_{h}^{q} f_{h}\right] P_{h}^{0} \varphi_{h}\right) \preccurlyeq \sum_{T \in \mathcal{T}_{h}} h_{T}^{2}\left\|f_{h}-I_{h}^{q} f_{h}\right\|_{\infty ; T}\|\varphi\|_{d /(d-2) ; \mathcal{U}_{h}(T)} \\
\leq & \leq\left[\sum_{T \in \mathcal{T}_{h}} h_{T}^{d}\left\|f_{h}-I_{h}^{q} f_{h}\right\|_{\infty ; T}^{d / 2}\right]^{2 / d}\|\varphi\|_{d /(d-2) ; \Omega} \preccurlyeq\left|\varepsilon_{d / 2}^{q}\right|_{d / 2}\left\|D^{2} \varphi\right\|_{1 ; \Omega},
\end{aligned}
$$

as a consequence of definition (3.11) of $\varepsilon_{d / 2}^{q}$ and the global Poincaré-type inequality $\|\varphi\|_{d /(d-2) ; \Omega} \preccurlyeq\left\|D^{2} \varphi\right\|_{1 ; \Omega}$. The latter follows from [12, Corollary 7.11] for $d>2$ and [6, Lemma 4.3.4] for $d=2$, after removing the lower order terms in $\|\varphi\|_{W_{1}^{2}(\Omega)}$ because $\varphi$ has zero trace.

For the second sum in (3.13), we use again quadrature stability (3.10a) to get

$$
E_{T}\left(\left[f_{h}-I_{h}^{q-1} f_{h}\right]\left[\varphi_{h}-P_{h}^{0} \varphi_{h}\right]\right) \preccurlyeq h_{T}^{d}\left\|f_{h}-I_{h}^{q-1} f_{h}\right\|_{\infty ; T}\left\|\varphi_{h}-P_{h}^{0} \varphi_{h}\right\|_{\infty ; T} .
$$

Proceeding as before, now with stability property $(3.3 \mathrm{~b})$ of $\Pi_{h}$, we obtain

$$
\begin{aligned}
\left\|\varphi_{h}-P_{h}^{0} \varphi_{h}\right\|_{\infty ; T} & \preccurlyeq h_{T}^{1-d}\left\|\varphi_{h}-P_{h}^{0} \varphi_{h}\right\|_{d /(d-1) ; T} \\
& \preccurlyeq h_{T}^{2-d}\left\|\nabla \varphi_{h}\right\|_{d /(d-1) ; T} \preccurlyeq h_{T}^{2-d}\|\nabla \varphi\|_{d /(d-1) ; \mathcal{U}_{h}(T)} .
\end{aligned}
$$

As above, we combine this with an Hölder inequality and the Poincaré-type inequality $\|\nabla \varphi\|_{d /(d-1) ; \Omega} \preccurlyeq\left\|D^{2} \varphi\right\|_{1 ; \Omega}[11$, Theorem 2 - p. 265] to write

$$
\begin{aligned}
\sum_{T \in \mathcal{T}_{h}} E_{T}\left(\left[f_{h}-I_{h}^{q-1} f_{h}\right]\left[\varphi_{h}-P_{h}^{0} \varphi_{h}\right]\right) \\
\quad \preccurlyeq \sum_{T \in \mathcal{T}_{h}} h_{T}^{2}\left\|f_{h}-I_{h}^{q-1} f_{h}\right\|_{\infty ; T}\|\nabla \varphi\|_{d /(d-1) ; \mathcal{U}_{h}(T)} \\
\quad \preccurlyeq\left[\sum_{T \in \mathcal{T}_{h}} h_{T}^{2 d}\left\|f_{h}-I_{h}^{q-1} f_{h}\right\|_{\infty ; T}^{d}\right]^{1 / d}\left\|D^{2} \varphi\right\|_{1 ; \Omega} \preccurlyeq\left|h \varepsilon_{d}^{q-1}\right|_{d}\left\|D^{2} \varphi\right\|_{1 ; \Omega} .
\end{aligned}
$$

Estimate (3.12a) is now a consequence of (3.13) combined with (3.14) and (3.15). 
We proceed similarly to show (3.12b), and only give the main steps. To estimate the first contribution in (3.13) we now use

$$
\left\|P_{h}^{0} \varphi_{h}\right\|_{\infty ; T} \preccurlyeq h_{T}^{1-d}\left\|\varphi_{h}\right\|_{d /(d-1) ; T} \preccurlyeq h_{T}^{1-d}\|\varphi\|_{d /(d-1) ; \mathcal{U}_{h}(T)},
$$

whence

$$
\begin{aligned}
\sum_{T \in \mathcal{T}_{h}} & E_{T}\left(\left[f_{h}-I_{h}^{q} f_{h}\right] P_{h}^{0} \varphi_{h}\right) \preccurlyeq \sum_{T \in \mathcal{T}_{h}} h_{T}\left\|f_{h}-I_{h}^{q} f_{h}\right\|_{\infty ; T}\|\varphi\|_{d /(d-1) ; \mathcal{U}_{h}(T)} \\
& \leq\left|h^{-1} \varepsilon_{d / 2}^{q}\right|_{d / 2 ; \Omega}\left[\sum_{T \in \mathcal{T}_{h}}\|\varphi\|_{d /(d-1) ; \mathcal{U}_{h}(T)}^{d /(d-2)}\right]^{(d-2) / d}=\left|h^{-1} \varepsilon_{d / 2}^{q}\right|_{d / 2 ; \Omega}\|\varphi\|_{d /(d-1) ; \Omega},
\end{aligned}
$$

because $|\xi|_{d /(d-2)} \leq|\xi|_{d /(d-1)}$ for all $\xi \in \mathbb{R}^{\# \mathcal{T}_{h}}$. The Poincaré-type inequality $\|\varphi\|_{d /(d-1) ; \Omega} \preccurlyeq\|\nabla \varphi\|_{p^{\prime} ; \Omega}$, valid for all $p^{\prime} \geq 1$, finally yields

$$
\sum_{T \in \mathcal{T}_{h}} E_{T}\left(\left[f_{h}-I_{h}^{q} f_{h}\right] P_{h}^{0} \varphi_{h}\right) \preccurlyeq\left|h^{-1} \varepsilon_{d / 2}^{q}\right|_{d / 2 ; \Omega}\|\nabla \varphi\|_{p^{\prime} ; \Omega} .
$$

For the second term in (3.13), we use

$$
\left\|\varphi_{h}-P_{h}^{0} \varphi_{h}\right\|_{\infty ; T} \preccurlyeq h_{T}^{1-d}\|\varphi\|_{d /(d-1) ; \mathcal{U}_{h}(T)}
$$

together with similar arguments to those above to arrive at

$$
\sum_{T \in \mathcal{T}_{h}} E_{T}\left(\left[f_{h}-I_{h}^{q-1} f_{h}\right]\left[\varphi_{h}-P_{h}^{0} \varphi_{h}\right]\right) \preccurlyeq\left|\varepsilon_{d}^{q-1}\right|_{d}\|\nabla \varphi\|_{p^{\prime} ; \Omega} .
$$

Estimate (3.12b) is then a consequence of (3.13), (3.16), and (3.17).

Combining the residual estimates (3.7) and quadrature estimates (3.12), in conjunction with (3.4), directly implies the following estimates for the negative norms

$$
\begin{aligned}
\|\psi\|_{-2, \infty ; \omega} & :=\sup \left\{\langle\psi, \varphi\rangle \mid \varphi \in \stackrel{\circ}{H}^{1}(\omega) \cap W_{1}^{2}(\omega),\left\|D^{2} \varphi\right\|_{1 ; \omega} \leq 1\right\}, \\
\|\psi\|_{-1, p ; \omega} & :=\sup \left\{\langle\psi, \varphi\rangle \mid \varphi \in \stackrel{\circ}{W}_{p^{\prime}}^{1}(\omega),\|\nabla \varphi\|_{p^{\prime} ; \omega} \leq 1\right\},
\end{aligned}
$$

defined for any open subset $\omega \subset \Omega, 1 \leq p \leq \infty$ and $p^{\prime}=p /(p-1)$, the dual exponent of $p$; the use of $\omega \subset \Omega$ will be essential in $\S 4.2$.

Corollary 3.4 (Control of the residual). Let the residual $\mathcal{R}_{h}$ be given by (3.1). Then, the following negative-norm estimates hold true

$$
\left\|\mathcal{R}_{h}\right\|_{-2, \infty ; \Omega} \preccurlyeq\left\|h^{2} R_{\infty}\right\|_{\infty ; \Omega}+\left|\varepsilon_{d / 2}^{q}\right|_{d / 2}+\left|h \varepsilon_{d}^{q-1}\right|_{d}
$$

and

$$
\left\|\mathcal{R}_{h}\right\|_{-1, p ; \Omega} \preccurlyeq\left\|h R_{p}\right\|_{\infty ; \Omega}+\left|h^{-1} \varepsilon_{d / 2}^{q}\right|_{d / 2}+\left|\varepsilon_{d}^{q-1}\right|_{d} .
$$

Remark 3.5 (Relation between residual and error). Having established in (3.18a) an estimate for the residual norm $\left\|\mathcal{R}_{h}\right\|_{-2, \infty ; \Omega}$, one might think that this would be equivalent to the pointwise error $\left\|u-u_{h}\right\|_{\infty ; \Omega}$. In $\S 4.2$ we will show

$$
\left\|u-u_{h}\right\|_{\infty ; \Omega} \preccurlyeq \eta_{h}
$$

where the error estimator $\eta_{h}$ contains the terms from the right hand side of (3.18a) plus the boundary correction term $\left\|g-g_{h}\right\|_{\infty ; \partial \Omega}$. Integration by parts in (3.1) yields the following identity for any function $\varphi \in W_{1}^{2}(\Omega) \cap \stackrel{\circ}{H}^{1}(\Omega)$

$$
\left\langle\mathcal{R}_{h}, \varphi\right\rangle=-\left\langle u-u_{h}, \Delta \varphi\right\rangle+\int_{\partial \Omega}\left(u-u_{h}\right) \partial_{\nu} \varphi+\left\langle f(\cdot, u)-f\left(\cdot, u_{h}\right), \varphi\right\rangle .
$$


Hence, we infer that for any subdomain $\omega \subset \Omega$

$$
\left\|\mathcal{R}_{h}\right\|_{-2, \infty ; \omega} \preccurlyeq\left\|u-u_{h}\right\|_{\infty ; \omega}+\left\|f(\cdot, u)-f\left(\cdot, u_{h}\right)\right\|_{-2, \infty ; \omega}
$$

holds just as well

$$
\left\|f(\cdot, u)-f\left(\cdot, u_{h}\right)\right\|_{-2, \infty ; \Omega} \preccurlyeq\left\|\mathcal{R}_{h}\right\|_{-2, \infty ; \Omega}+\left\|u-u_{h}\right\|_{\infty ; \Omega} \preccurlyeq \eta_{h} .
$$

From these estimates we see that error $\left\|u-u_{h}\right\|_{\infty ; \Omega}$ and residual $\left\|\mathcal{R}_{h}\right\|_{-2, \infty ; \Omega}$ do not relate directly. For $\left\|\mathcal{R}_{h}\right\|_{-2, \infty ; \Omega}$ to be an effective error measure we need to enlarge the error concept and incorporate $\left\|f(\cdot, u)-f\left(\cdot, u_{h}\right)\right\|_{-2, \infty ; \Omega}$.

Remark 3.6 (Order of consistency estimators). The proof of Lemma 3.2 only requires $q \geq \ell$. However, since $q \geq \max \{2 \ell-2,1\}>\ell$ for $\ell>2$ and interpolation operators of order $q$ and $q-1$ occur in the definitions of $\varepsilon_{d / 2}^{q}$ and $\varepsilon_{d}^{q-1}$, we may wonder how our a posteriori error analysis compares with the well established a priori error analysis [9, Section 4.1]. We first note that the test for the residual $\mathcal{R}_{h}$ in (3.4) involves only piecewise linear polynomials rather than polynomials of degree $\leq \ell$. This allows us to exploit more cancellation in (3.13) than it is possible in the a priori analysis. For smooth functions $f(x, u)$, the consistency estimators may decay faster than the residual estimator $\left\|h^{2} R_{\infty}\right\|_{\infty ; \Omega}$. On the other hand for rough functions this may not be the case; we explore this issue further in Remark 4.4. In any event, it is the collective contribution of both residual and consistency estimators what controls $\left\|\mathcal{R}_{h}\right\|_{-2, \infty ; \Omega}$ and eventually the pointwise error.

3.3. Estimating the Riesz representation. In this section, we use (3.18) in order to prove an a posteriori $L_{\infty}$ estimate for the Riesz projection $w=(-\Delta)^{-1} \mathcal{R}_{h}$. We establish this bound in three steps: We first bound $w$ in a Hölder space, thus stronger than $L_{\infty}(\Omega)$. We then give an estimate weaker than $\|w\|_{\infty ; \Omega}$, and finally we combine these two estimates. We use the same techniques as in [19] but, since they are not standard, we repeat them here for the reader's convenience.

Lemma 3.7 (Hölder continuity of $w$ and its control). The Riesz representation $w$ of the residual $\mathcal{R}_{h}$ is Hölder continuous. More precisely, for every $p>d$ there exists $\alpha \in(0,1)$ such that

$$
\|w\|_{C^{0, \alpha}(\bar{\Omega})} \leq C\left\|\mathcal{R}_{h}\right\|_{-1, p ; \Omega},
$$

where the constant $C$ depends on $\Omega$ and $p$, and blows up as $p \downarrow d$.

Proof. Estimate (3.21) is a classical Hölder estimate of De Giorgi and Nash (see e.g. [15, Theorem C.2]).

Recalling estimate $(3.18 \mathrm{~b})$ for $\left\|\mathcal{R}_{h}\right\|_{-1, p ; \Omega}$ we realize that the right hand side of (3.21) is a first order estimator only, while we have to estimate $\|w\|_{\infty ; \Omega}$ by a second order estimator (assuming a smooth nonlinearity $f$ ). A key step for recovering a second order estimator is performed next.

Since $w$ is continuous and satisfies $w_{\mid \partial \Omega}=0$, there exists a point $x_{0} \in \Omega$ with $\left|w\left(x_{0}\right)\right|=\|w\|_{\infty ; \Omega}$. Invoking the uniform cone property of $\Omega$ [1, Section 4.7], we can choose a ball $B$ with radius $\rho$ such that $B \subset \Omega$, $\operatorname{dist}\left(x_{0}, B\right) \preccurlyeq \rho$, and $\rho=C h_{\text {min }}^{\beta}$, where $h_{\min }$ is the minimal meshsize of $\mathcal{T}_{h}$, i. e.

$$
h_{\min }=\min _{T \in \mathcal{T}_{h}} h_{T}
$$


and $\beta>1$ will be chosen later. Now, let $\delta \in C_{0}^{\infty}(\Omega)$ be a regularization of the Dirac mass satisfying

$$
\operatorname{supp} \delta \subset B, \quad \int_{\Omega} \delta=1, \quad 0 \leq \delta \preccurlyeq \rho^{-d} .
$$

Taking $x_{1} \in B$ such that $\langle\delta, w\rangle=w\left(x_{1}\right)$, we may write

$$
\|w\|_{\infty ; \Omega} \leq|\langle\delta, w\rangle|+\left|w\left(x_{0}\right)-w\left(x_{1}\right)\right| .
$$

Lemma 3.7 implies the following estimate for the second term of (3.23):

$$
\left|w\left(x_{0}\right)-w\left(x_{1}\right)\right| \preccurlyeq h_{\min }^{\alpha \beta}\left\|\mathcal{R}_{h}\right\|_{-1, p ; \Omega} .
$$

A bound for the first term in (3.23) is established in the next lemma.

Lemma 3.8 (Estimate for $\langle\delta, w\rangle)$. The regularized Dirac mass $\delta$ of (3.22) and the Riesz representation $w$ of the residual $\mathcal{R}_{h}$ satisfy

$$
|\langle\delta, w\rangle| \leq C\left|\log h_{\min }\right|^{2}\left\|\mathcal{R}_{h}\right\|_{-2, \infty ; \Omega},
$$

where the geometric constant $C$ depends on $\beta$ via $\rho$.

Proof. Introducing the regularized Green's function $G \in H^{1}(\Omega)$ defined by

$$
\langle\nabla G, \nabla \varphi\rangle=\langle\delta, \varphi\rangle \quad \text { for all } \varphi \in \stackrel{\circ}{H}^{1}(\Omega),
$$

we obtain in light of $(3.2)$

$$
|\langle\delta, w\rangle|=|\langle\nabla G, \nabla w\rangle|=\left|\left\langle\mathcal{R}_{h}, G\right\rangle\right| \preccurlyeq\left\|\mathcal{R}_{h}\right\|_{-2, \infty ; \Omega}\left\|D^{2} G\right\|_{1 ; \Omega} .
$$

The assertion of the lemma now follows by applying the following estimate of Nochetto [17] in two and of Dari et al. [10] in three space dimensions for the second derivatives of the regularized Green's function in any polyhedral domain:

$$
\left\|D^{2} G\right\|_{1 ; \Omega} \preccurlyeq\left|\log h_{\min }\right|^{2} .
$$

The constant hidden in $\preccurlyeq$ depends on $\beta$ via $\rho$.

Combining the two previous lemmas yields the main result of this section.

Proposition 3.9 (Pointwise estimate of $|w|$ ). The maximum norm of the Riesz representation $w$ of the residual $\mathcal{R}_{h}$ satisfies the a posteriori bound

$$
\|w\|_{\infty ; \Omega} \preccurlyeq\left|\log h_{\text {min }}\right|^{2}\left[\left\|h^{2} R_{\infty}\right\|_{\infty ; \Omega}+\left|\varepsilon_{d / 2}^{q}\right|_{d / 2}+\left|h \varepsilon_{d}^{q-1}\right|_{d}\right] .
$$

Proof. Combining (3.23) and (3.24) together with Lemma 3.8 yields

$$
\|w\|_{\infty ; \Omega} \preccurlyeq\left|\log h_{\min }\right|^{2}\left\|\mathcal{R}_{h}\right\|_{-2, \infty ; \Omega}+h_{\min }^{\alpha \beta}\left\|\mathcal{R}_{h}\right\|_{-1, p ; \Omega} .
$$

Estimate (3.18a)

$$
\left\|\mathcal{R}_{h}\right\|_{-2, \infty ; \Omega} \preccurlyeq\left\|h^{2} R_{\infty}\right\|_{\infty ; \Omega}+\left|\varepsilon_{d / 2}^{q}\right|_{d / 2}+\left|h \varepsilon_{d}^{q-1}\right|_{d}
$$

directly gives the desired second order estimator for the first term of the right hand side of (3.26).

Let $p>d$ be fixed and let $\alpha \in(0,1)$ be given by Lemma 3.7. Now choosing $\beta=1 / \alpha$, we see $h_{\text {min }}^{\alpha \beta}=h_{\min } \leq h_{T}$ for all $T \in \mathcal{T}_{h}$ and, recalling (3.18b), we achieve the second order estimator also for the second term of (3.26)

$$
\begin{aligned}
h_{\min }^{\alpha \beta}\left\|\mathcal{R}_{h}\right\|_{-1, p ; \Omega} & \preccurlyeq h_{\min }\left[\left\|h R_{p}\right\|_{\infty ; \Omega}+\left|h^{-1} \varepsilon_{d / 2}^{q}\right|_{d / 2}+\left|\varepsilon_{d}^{q-1}\right|_{d}\right] \\
& \preccurlyeq\left\|h^{2} R_{\infty}\right\|_{\infty ; \Omega}+\left|\varepsilon_{d / 2}^{q}\right|_{d / 2}+\left|h \varepsilon_{d}^{q-1}\right|_{d},
\end{aligned}
$$


where we have used a Hölder inequality for the first term.

\section{A Posteriori Error Estimates}

The a posteriori upper bound for the pointwise error $\left\|u-u_{h}\right\|_{\infty ; \Omega}$ is established in two steps. In the first step, the Riesz projection $w$ of the residual $\mathcal{R}_{h}$ is used for constructing barrier functions for the true solution. These barrier functions together with the pointwise estimate (3.25) of $w$ then directly yield the upper bound.

4.1. Barrier functions for the true solution. The basic idea for the construction of the barrier functions for the true solution is a correction of the discrete solution $u_{h}$ by means of $w$ and a term due to approximation of boundary values.

Proposition 4.1 (Upper and lower barriers). Let $u_{h}$ be the discrete solution given by (2.4), $w$ be the Riesz representation (3.2) of the residual $\mathcal{R}_{h}$, and $g_{h}$ be an approximation of boundary data $g$. Then, the functions

$$
u^{*}:=u_{h}+w+\|w\|_{\infty ; \Omega}+\left\|g-g_{h}\right\|_{\infty ; \partial \Omega}
$$

and

$$
u_{*}:=u_{h}+w-\|w\|_{\infty ; \Omega}-\left\|g-g_{h}\right\|_{\infty ; \partial \Omega}
$$

are an upper, respectively lower barrier to the true solution $u$ of $(2.1)$, i. e.

$$
u_{*} \leq u \leq u^{*} \quad \text { in } \Omega .
$$

Proof. To establish that $u^{*}$ is an upper barrier of $u$, we let $v=\left(u-u^{*}\right)_{+}=$ $\max \left\{u-u^{*}, 0\right\}$ be the non-negative part of $u-u^{*}$. On $\partial \Omega$, we have

$$
u-u_{h}-w-\|w\|_{\infty ; \Omega}-\left\|g-g_{h}\right\|_{\infty ; \partial \Omega} \leq g-g_{h}-\left\|g-g_{h}\right\|_{\infty ; \partial \Omega} \leq 0,
$$

whence $v=0$ on $\partial \Omega$. By the definition (3.1) of the residual $\mathcal{R}_{h}$ we obtain

$$
\begin{aligned}
\|\nabla v\|_{2 ; \Omega}^{2} & =\left\langle\nabla\left(u-u^{*}\right), \nabla v\right\rangle=\left\langle\nabla\left(u-u_{h}\right), \nabla v\right\rangle-\langle\nabla w, \nabla v\rangle \\
& =\left\langle\nabla\left(u-u_{h}\right), \nabla v\right\rangle-\left\langle\mathcal{R}_{h}, v\right\rangle=-\left\langle f(\cdot, u)-f\left(\cdot, u_{h}\right), v\right\rangle \\
& =-\int_{\{v>0\}}\left[f(\cdot, u)-f\left(\cdot, u_{h}\right)\right] v d x .
\end{aligned}
$$

Let $x \in \Omega$ with $v(x)>0$. This implies $u(x)>u^{*}(x)$ which gives

$$
u(x)>u_{h}(x)+\underbrace{w(x)+\|w\|_{\infty ; \Omega}}_{\geq 0}+\left\|g-g_{h}\right\|_{\infty ; \partial \Omega} \geq u_{h}(x) .
$$

The monotonicity of $f$ in the second argument then yields $f(x, u(x)) \geq f\left(x, u_{h}(x)\right)$ for $v(x)>0$ whence $\left[f(x, u(x))-f\left(x, u_{h}(x)\right)\right] v(x) \geq 0$. Therefore

$$
\|\nabla v\|_{2 ; \Omega}^{2} \leq-\int_{\{v>0\}}\left[f(x, u(x))-f\left(x, u_{h}(x)\right)\right] v(x) d x \leq 0
$$

which implies $v \equiv 0$ since $v \equiv 0$ on $\partial \Omega$. Hence, we derived the upper bound $u \leq u^{*}$.

To establish that $u_{*}$ is a lower barrier of $u$, we now use $v=\left(u_{*}-u\right)_{+}=$ $\max \left\{u_{*}-u, 0\right\}$. As above $v=0$ holds on $\partial \Omega$, thanks to the correction term $-\left\|g-g_{h}\right\|_{\infty ; \partial \Omega}$ in the definition of $u_{*}$. Definition (3.1) of the residual $\mathcal{R}_{h}$ then gives

$$
\|\nabla v\|_{2 ; \Omega}^{2}=\left\langle f(\cdot, u)-f\left(\cdot, u_{h}\right), v\right\rangle=\int_{\{v>0\}}\left[f(\cdot, u)-f\left(\cdot, u_{h}\right)\right] v d x .
$$


For points $x \in \Omega$ with $v(x)>0$ we conclude

$$
u(x)<u_{h}(x)+w(x)-\|w\|_{\infty ; \Omega}-\left\|g-g_{h}\right\|_{\infty ; \partial \Omega} \leq u_{h}(x) .
$$

Monotonicity of $f$ implies $f(x, u(x)) \leq f\left(x, u_{h}(x)\right)$ for $v(x)>0$, which yields

$$
\|\nabla v\|_{2 ; \Omega}^{2} \leq \int_{\{v>0\}}\left[f(x, u(x))-f\left(x, u_{h}(x)\right)\right] v(x) d x \leq 0
$$

and thus $u_{*} \leq u$. This is the asserted lower bound.

4.2. Error estimator: Pointwise upper and lower bounds. A consequence of the barriers (4.1) is the bound

$$
\left\|u-u_{h}\right\|_{\infty ; \Omega} \leq 2\|w\|_{\infty ; \Omega}+\left\|g-g_{h}\right\|_{\infty ; \partial \Omega} .
$$

Pointwise control (3.25) of $w$ in terms of the discrete solution and given data now directly gives rise to the a posteriori error estimator

$$
\eta_{h}=\left|\log h_{\min }\right|^{2}\left[c_{0}\left\|h^{2} R_{\infty}\right\|_{\infty ; \Omega}+c_{1}\left|\varepsilon_{d / 2}^{q}\right|_{d / 2}+c_{2}\left|h \varepsilon_{d}^{q-1}\right|_{d}\right]+\left\|g-g_{h}\right\|_{\infty ; \partial \Omega} .
$$

Here $h_{\min }$ is the smallest meshsize, $R_{\infty}$ is the local residual, defined in (3.6), $q$ is the quadrature order $(2.3), \varepsilon_{p}^{j}$ is the local consistency estimate, defined in (3.11), and $g_{h}=I_{h} g$ is the approximation of boundary data in $\mathbb{V}_{h}$. Finally, $c_{0}, c_{1}, c_{2}$ are the constants appearing in the analysis presented above, and may depend on the degree $q$ of quadrature formula, the shape regularity of the underlying triangulation $\mathcal{T}_{h}$, and $\Omega$. Remarkably, these constants do not depend on the nonlinearilty $f$, which is assumed to be continuous in both arguments and monotone in the second one.

Theorem 4.2 (Reliability). Let $u$ be the true solution given by (2.1) and $u_{h}$ the discrete solution given by (2.4). Then the following a posteriori estimates hold

$$
\left\|u-u_{h}\right\|_{\infty ; \Omega} \leq \eta_{h}, \quad\left\|f(\cdot, u)-f\left(\cdot, u_{h}\right)\right\|_{-2, \infty ; \Omega} \preccurlyeq \eta_{h} .
$$

Proof. The estimate for $\left\|u-u_{h}\right\|_{\infty ; \Omega}$ is obvious in view of (3.25) and (4.2). The estimate for $\left\|f(\cdot, u)-f\left(\cdot, u_{h}\right)\right\|_{-2, \infty ; \Omega}$ then directly follows from estimate $(3.20)$ in Remark 3.5.

Finally, the lower bounds are proven with the standard techniques introduced by Verfürth [26]. We also refer to $[17,19]$ for the $L_{\infty}$ analysis. We sketch the proofs and start by defining data oscillation as follows:

$$
\operatorname{osc}\left(f_{h} ; \omega\right):=\left\|h^{2}\left[f\left(\cdot, u_{h}\right)-\bar{f}\left(\cdot, u_{h}\right)\right]\right\|_{\infty ; \omega},
$$

where $\omega$ is a union of elements and $\bar{f}$ is a piecewise polynomial approximation of $f$ of degree $\geq q+1$ so that $\operatorname{osc}\left(f_{h} ; \omega\right)$ is formally of higher order than any contribution in $\eta_{h}$. We say that a local (global) indicator is locally (globally) efficient if it is dominated by the local (global) error plus local (global) data oscillation.

Theorem 4.3 (Local efficiency). The residual indicator $\left\|h^{2} R_{\infty}\right\|_{\infty ; \Omega}$ and boundary datum indicator $\left\|g-g_{h}\right\|_{\infty ; \partial \Omega}$ are locally efficient. In particular, for all $T \in \mathcal{T}_{h}$ the bound

$$
\begin{aligned}
\left\|h^{2} R_{\infty}\right\|_{\infty ; T} & +\left\|g-g_{h}\right\|_{\infty ; \partial T \cap \partial \Omega} \\
& \preccurlyeq\left\|u-u_{h}\right\|_{\infty ; \omega(T)}+\left\|f(\cdot, u)-f\left(\cdot, u_{h}\right)\right\|_{-2, \infty ; \omega(T)}+\operatorname{osc}\left(f_{h} ; \omega(T)\right)
\end{aligned}
$$


is valid, where $\omega(T)$ is the union of all simplices in $\mathcal{T}_{h}$ that share one side with $T$. On the other hand, if $q \geq \ell-1$, the consistency indicators are locally efficient, namely

$$
\varepsilon_{d / 2, T}^{q}+h_{T} \varepsilon_{d, T}^{q-1} \preccurlyeq\left\|u-u_{h}\right\|_{\infty ; \omega(T)}+\left\|f(\cdot, u)-f\left(\cdot, u_{h}\right)\right\|_{-2, \infty ; \omega(T)}+\operatorname{osc}\left(f_{h} ; \omega(T)\right) .
$$

Proof. Since $g-g_{h}=u-u_{h}$, the estimate for the boundary data indicator is trivial.

We now recall the definition (3.6) of $\left.R_{\infty}\right|_{T}$ for $T \in \mathcal{T}_{h}$, and first deal with the interior indicator. We again set $f_{h}=f\left(\cdot, u_{h}\right)$ and define $\bar{f}_{h}=\bar{f}\left(\cdot, u_{h}\right)$. Since $\left.\left(\Delta u_{h}-\bar{f}_{h}\right)\right|_{T}$ is a polynomial of degree $k \geq q+1$, we have

$$
\left\|\Delta u_{h}-\bar{f}_{h}\right\|_{\infty ; T} \preccurlyeq \int_{T}\left(\Delta u_{h}-\bar{f}_{h}\right) \zeta_{T} b_{T}
$$

for a suitable $\zeta_{T} \in \mathbb{P}_{k}(T)$ with $\left\|\zeta_{T}\right\|_{1 ; T} \leq 1$; here $b_{T}$ is a suitable bubble function in $W_{1}^{2}(T) \cap \stackrel{\circ}{H}^{1}(T)$; see $[17,19,26]$. Integration by parts and $(2.1)$ yield

$$
\int_{T}\left(\Delta u_{h}-\bar{f}_{h}\right) \zeta_{T} b_{T}=\left\langle\mathcal{R}_{h}, \zeta_{T} b_{T}\right\rangle+\int_{T}\left(f_{h}-\bar{f}_{h}\right) \zeta_{T} b_{T}
$$

whence, with the aid of $\left\|\zeta_{T} b_{T}\right\|_{2,1: T} \preccurlyeq\left\|D^{2}\left(\zeta_{T} b_{T}\right)\right\|_{1, T} \preccurlyeq h_{T}^{-2}$ and (3.19), we deduce

$$
h_{T}^{2}\left\|\Delta u_{h}-f_{h}\right\|_{\infty ; T} \preccurlyeq\left\|u-u_{h}\right\|_{\infty ; T}+\left\|f-f_{h}\right\|_{-2, \infty ; T}+\operatorname{osc}\left(f_{h} ; T\right) .
$$

We consider next the jump residual. Given a side $S$, let $b_{S}$ be a suitable bubble function in $W_{1}^{2}(\omega(S)) \cap \stackrel{\circ}{H}^{1}(\omega(S))$, where $\omega(S)$ is the union of the two elements sharing $S$; see [19]. Since $\llbracket \partial_{n} u_{h} \rrbracket \in \mathbb{P}_{\ell-1}(S)$, for a suitable function $\zeta_{S} \in \mathbb{P}_{\ell-1}(\omega(S))$ such that $\left\|\zeta_{S}\right\|_{1 ; S} \preccurlyeq 1$, we have after integration by parts

$$
\left\|\llbracket \partial_{n} u_{h} \rrbracket\right\|_{\infty ; S} \preccurlyeq \int_{S} \llbracket \partial_{n} u_{h} \rrbracket \zeta_{S} b_{S}=\left\langle\mathcal{R}_{h}, \zeta_{S} b_{S}\right\rangle+\left\langle f_{h}-\Delta u_{h}, \zeta_{S} b_{S}\right\rangle,
$$

whence

$$
\left\|\llbracket \partial_{n} u_{h} \rrbracket\right\|_{\infty ; S} \preccurlyeq h_{S}^{-1}\left\|\mathcal{R}_{h}\right\|_{-2, \infty ; \omega(S)}+h_{S}\left\|f_{h}-\Delta u_{h}\right\|_{\infty ; \omega(S)} .
$$

Combining this with (3.19) and the previous estimate for the interior residual, we obtain the desired local estimate for $\left\|h^{2} R_{\infty}\right\|_{\infty ; T}$.

We finally deal with the consistency indicators. We note first that

$$
\varepsilon_{d / 2, T}^{q} \preccurlyeq h_{T}^{2}\left\|f_{h}-I_{h}^{q} f_{h}\right\|_{\infty ; T} \preccurlyeq h_{T}^{2}\left\|f_{h}-\bar{f}_{h}\right\|_{\infty ; T}+h_{T}^{2}\left\|\bar{f}_{h}-I_{h}^{q} f_{h}\right\|_{\infty ; T}
$$

Since $\bar{f}_{h}-I_{h}^{q} f_{h}$ is a polynomial in $T$, there exists a polynomial $\zeta_{T}$ of the same degree with $\left\|\zeta_{T}\right\|_{1 ; T} \preccurlyeq 1$ such that

$$
\begin{aligned}
\left\|\bar{f}_{h}-I_{h}^{q} f_{h}\right\|_{\infty ; T} & \preccurlyeq \int_{T}\left(\bar{f}_{h}-I_{h}^{q} f_{h}\right) \zeta_{T} b_{T} \\
& =\int_{T}\left(\left(f_{h}-\Delta u_{h}\right)-I_{h}^{q}\left(f_{h}-\Delta u_{h}\right)\right) \zeta_{T} b_{T}+\int_{T}\left(\bar{f}_{h}-f_{h}\right) \zeta_{T} b_{T}
\end{aligned}
$$

because $\Delta u_{h} \in \mathbb{P}_{\ell-2}(T)$ and $q \geq \ell-2$. Consequently, we conclude

$$
h_{T}^{2}\left\|f_{h}-I_{h}^{q} f_{h}\right\|_{\infty ; T} \preccurlyeq\left\|h^{2} R_{\infty}\right\|_{\infty ; T}+\operatorname{osc}\left(f_{h} ; T\right)
$$

and thus the asserted estimate for $\varepsilon_{d / 2, T}^{q}$ is established. We next observe that $h_{T} \varepsilon_{d, T}^{q-1} \preccurlyeq h_{T}^{2}\left\|f_{h}-I_{h}^{q-1} f_{h}\right\|_{\infty ; T}$, and so the same argument as of $\varepsilon_{d / 2, T}^{q}$ applies whenever $q-1 \geq \ell-2$. This completes the proof. 
Remark 4.4 (Global efficiency). The local bounds for the indicators $\left\|h^{2} R_{\infty}\right\|_{\infty ; T}$ and $\left\|g-g_{h}\right\|_{\infty ; \partial T \cap \partial \Omega}$ in Theorem 4.3 immediately imply a corresponding global lower bound and so these estimator contributions are globally efficient too. This is not clear for the consistency indicators $\varepsilon_{d / 2, T}^{q}$ and $h_{T} \varepsilon_{d, T}^{q-1}$, since they accumulate differently from the maximum norm error: contrast

$$
\left|\varepsilon_{p}^{j}\right|_{p}^{p}=\sum_{T \in \mathcal{T}_{h}}\left(\varepsilon_{p, T}^{j}\right)^{p}=\sum_{T \in \mathcal{T}_{h}}|T|\left\|f_{h}-I_{h}^{j} f_{h}\right\|_{\infty ; T}^{p} .
$$

with

$$
\left\|u-u_{h}\right\|_{\infty ; \Omega}=\max _{T \in \mathcal{T}_{h}}\left\|u-u_{h}\right\|_{\infty ; \omega(T)} .
$$
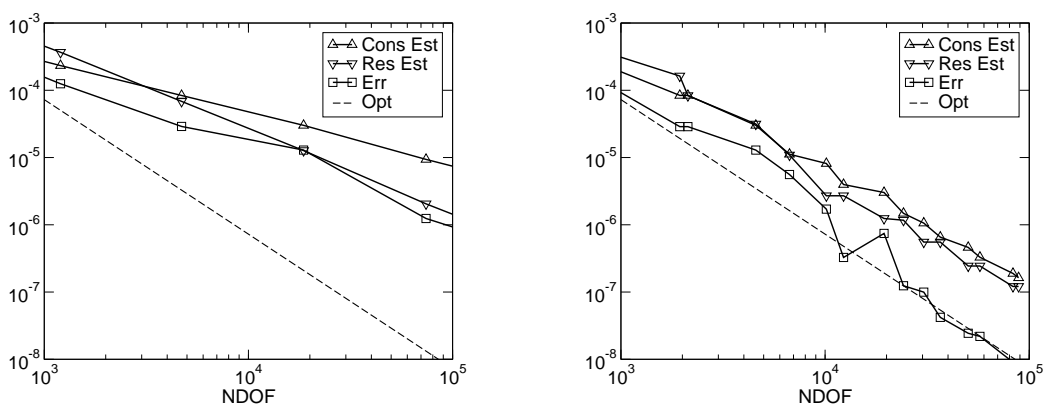

Figure 4.1. Decay of error and estimators (residual and consistency parts) vs. number of degrees of freedom (NDOF) for a linear problem with non-smooth right hand side: uniform refinement (left) and adaptive refinement (right).

In order to investigate this issue, which is unrelated to the nonlinear structure of (1.1), we numerically compare the computable part of the error $\left\|u-u_{h}\right\|_{\infty ; \Omega}$ and the two estimator contributions $\left\|h^{2} R_{\infty}\right\|_{\infty ; \Omega}+\left\|g-g_{h}\right\|_{\infty ; \partial \Omega}$ and $\left|\varepsilon_{d / 2}^{q}\right|_{d / 2}+\left|h \varepsilon_{d}^{q-1}\right|_{d}$ for the linear Poisson equation

$$
-\Delta u=f \quad \text { in } \Omega, \quad u=g \quad \text { on } \partial \Omega .
$$

For smooth right hand sides $f$, numerical experiments suggest that the local consistency indicators, which read $|T|^{1 / p}\left\|f-I_{h}^{j} f\right\|_{\infty ; T}$ for the linear problem, exhibit enough local cancellations for an optimal decay of the global consistency estimates $\left|\varepsilon_{p}^{j}\right|_{p}$. This is also observed for the nonlinear problem; see $\S 5.2$.

For the discussion on nonsmooth data, consider now the exact solution $u(x)=$ $(|x|-R)_{+}^{\alpha}$ with the corresponding forcing function

$$
f(x)=\alpha\left(\alpha-1+\frac{(|x|-R)_{+}}{|x|}\right)(|x|-R)_{+}^{\alpha-2}
$$

with $\alpha=2.5, R=0.5$ and $\Omega=(0,1)^{2}$; the solution $u$ is related to the semilinear example of $\S 5.3$. Since $f$ is Hölder continuous with exponent $1 / 2$ but not $C^{1}$, the effect of quadrature is noticeable for all polynomial degree $\ell \geq 1$. Figure 4.1 depicts the results for the case of uniform and adaptive refinement, using the marking strategy designed in $\S 5.1$, with $\ell=3$ and $q=4$ for $d=2$. It suggests that:

- the quadrature indicators are not globally efficient in general; 
- for this particular example, the convergence rate of the estimator is slightly less than the rate $(\ell+1) / d=2$ for regular forcing functions $f$, thereby indicating a suboptimal decay for non-smooth data due to the missing global efficiency of the quadrature indicators;

- in spite of the preceding observation, adaptive refinement is still far superior to uniform one.

Remark 4.5 (Efficient consistency control). As already observed in [19], the quantity $\left\|\mathcal{E}_{h}\right\|_{-2, \infty ; \Omega}$ is globally efficient. In fact, combining the relation of residual and consistency error (3.4) with the bound for the residual (3.19) yields

$$
\left\|\mathcal{E}_{h}\right\|_{-2, \infty ; \Omega} \preccurlyeq\left\|u-u_{h}\right\|_{\infty ; \Omega}+\left\|f-f_{h}\right\|_{-2, \infty ; \Omega} .
$$

However, $\left\|\mathcal{E}_{h}\right\|_{-2, \infty ; \Omega}$ is a global and noncomputable quantity and thus of little practical value. Due to the product structure of the cancellation in $\mathcal{E}_{h}$, we are forced to deal with Sobolev spaces $L_{d}(\Omega)$ and $W_{d / 2}^{1}(\Omega)$ instead of $W_{1}^{2}(\Omega)$ in the derivation of local and computable indicators. We conclude that the efficient control of quadrature deserves further investigation even for linear PDEs.

\section{NUMERICAL EXPERIMENTS}

We have implemented the nonlinear solver, error estimator and corresponding marking strategy using the adaptive finite element package $\operatorname{ALBERTA}^{1}[24,25]$. In ALBERTA, an initial simplicial macro-mesh is refined by successive bisections of its elements. Moreover, it can later be coarsened, by operations of junction of two elements which initially constituted a single element.

The discrete nonlinear problems are solved with a damped Newton iteration. The resulting linearized equations are solved by a preconditioned CG method. For the experiments shown below, the Newton solver converged quite well with only few damping steps.

5.1. Marking strategy. The aim of adaptive finite element methods (AFEM) is the generation of a sequence of meshes by local refinement such that eventually the error is below a given tolerance whereas the number of degrees of freedom (NDOF) used for the finite element solution is as small as possible.

We follow the common, but still heuristic, practice of marking elements with relatively large error indicators [5]; in general, this leads to quasi-optimal meshes, a crucial property that has not yet been proved theoretically. In the present case, however, the error estimator consists of three terms with different accumulation properties:

$$
\begin{aligned}
\eta_{\infty} & :=c_{0}\left\|h^{2} R_{\infty}\right\|_{\infty ; \Omega}+\left\|g-g_{h}\right\|_{\infty ; \partial \Omega}, \\
\eta_{d / 2} & :=c_{1}\left|\varepsilon_{d / 2}^{q}\right|_{d / 2}, \\
\eta_{d} & :=c_{2}\left|h \varepsilon_{d}^{q-1}\right|_{d} .
\end{aligned}
$$

A marking strategy aiming at quasi-optimal meshes must account for this fact. Similarly to [19], we proceed in two steps:

\footnotetext{
${ }^{1}$ The original name of the toolbox ALBERT had to be renamed due to copyright reasons.
} 
- We select an estimator $\eta_{p}, p \in\{d / 2, d, \infty\}$, solely whenever it is relatively large with respect to the total estimate, and thus its role is expected to be significant. We implement this idea by first computing

$$
\eta_{\max }=\max \left\{\eta_{\infty}, \eta_{d / 2}, \eta_{d}\right\}
$$

and then choosing $\eta_{p}$ provided $\eta_{p} \geq \bar{\theta} \eta_{\max }$, where $0<\bar{\theta}<1$ is a given parameter.

- For each $\eta_{p}$ so chosen, we use the maximum strategy to mark elements $\hat{T} \in \mathcal{T}_{h}$ such that

$$
\eta_{p}(\hat{T}) \geq \theta \max _{T \in \mathcal{T}_{h}} \eta_{p}(T)
$$

where $0<\theta<1$ is a given parameter and $\eta_{p}(T)$ is the element indicator.

In the experiments below, we have neglected the logarithmic factor $\left|\log h_{\min }^{2}\right|^{2}$ in the estimator and used, instead, $\tilde{\eta}=\eta_{\infty}+\eta_{d / 2}+\eta_{d}$. The $L_{\infty}$-norms of element indicators are approximated by computing the maximum absolute value among all Lagrange nodes for 7 th order polynomials. Parameters for the marking strategy were $\bar{\theta}=0.7$ in selecting contributions and $\theta=0.5$ for the standard maximum strategy.

\begin{tabular}{|r||r|r|r||r|r|r||r|r|r|}
\hline \multicolumn{1}{|c||}{} & \multicolumn{3}{|c||}{$\mathbb{P}_{1}$} & \multicolumn{3}{|c||}{$\mathbb{P}_{2}$} & \multicolumn{3}{|c|}{$\mathbb{P}_{3}$} \\
\hline Iter & $\mathrm{NE}$ & $\mathrm{NR}$ & $\mathrm{NC}$ & $\mathrm{NE}$ & $\mathrm{NR}$ & $\mathrm{NC}$ & $\mathrm{NE}$ & $\mathrm{NR}$ & $\mathrm{NC}$ \\
\hline 1 & 4 & 4 & 0 & 4 & 4 & 0 & 4 & 4 & 0 \\
2 & 16 & 16 & 0 & 16 & 16 & 0 & 16 & 14 & 0 \\
3 & 64 & 48 & 0 & 64 & 52 & 0 & 62 & 30 & 0 \\
4 & 222 & 170 & 0 & 240 & 10 & 0 & 178 & 62 & 12 \\
5 & 766 & 464 & 0 & 276 & 86 & 20 & 420 & 12 & 0 \\
6 & 2332 & 472 & 0 & 592 & 186 & 0 & 460 & 148 & 16 \\
7 & 4108 & 2626 & 6 & 1246 & 0 & 38 & 1044 & 40 & 56 \\
8 & 12806 & 1036 & 10 & 1456 & 470 & 80 & 1532 & 0 & 10 \\
9 & 16792 & 9806 & 14 & 3526 & 474 & 18 & 1602 & 0 & 86 \\
10 & 48812 & 0 & 30 & 5348 & 0 & 104 & 2208 & 136 & 4 \\
11 & 49006 & 4998 & 48 & 5994 & 506 & 56 & 2760 & 0 & 24 \\
12 & 68284 & 0 & 18 & 8394 & 3234 & 132 & 2952 & 0 & 124 \\
13 & 68420 & 37698 & 58 & 20358 & 0 & 100 & 3954 & 248 & 36 \\
14 & 188980 & 0 & 32 & 21136 & 682 & 60 & 5322 & 0 & 76 \\
15 & 189194 & 0 & 86 & 24420 & 0 & 338 & 5962 & 0 & 282 \\
16 & 189774 & 19666 & 128 & 26698 & 4472 & 168 & 8162 & 262 & 22 \\
\hline
\end{tabular}

TABLE 5.1. Free boundary problem $(d=2)$ : Behavior of the marking strategy for the first 16 iterations of AFEM. NE is the number of mesh elements, and NR, NC are the number of elements marked due to residual indicator and consistency indicator, for piecewise linear $\mathbb{P}_{1}$, quadratic $\mathbb{P}_{2}$, and cubic $\mathbb{P}_{3}$ finite elements. The role of quadrature is more pronounced for higher order elements because the residuals decay faster.

Table 5.1 shows results of the marking strategy for piecewise linear $\mathbb{P}_{1}$, quadratic $\mathbb{P}_{2}$, and cubic $\mathbb{P}_{3}$ finite elements for the free boundary problem of Section 5.3 in the case $d=2$. It records, for each iteration of our AFEM, the current number of 
mesh elements NE, the number NR of elements marked due to residual indicator, and the number $\mathrm{NC}$ of elements marked due to consistency estimate. After the residuals get smaller during the first iterations, the consistency indicators cause some additional mesh refinements. Consistency marking is more pronounced for higher order approximations because the residuals decay faster. The effect of the consistency estimator is even more noticeable in the boundary layer example of Section 5.4, where the nonlinearity blows up and dominates the computation. After a few iterations, nearly all refinement is due to consistency indicator.

5.2. Smooth nonlinearity. The nonlinear stationary Poisson-Boltzmann equation for the potential $u$ corresponding to a given charge density $\rho(x)$ reads

$$
-\Delta u+\kappa^{2} \sinh (u)=\rho(x) .
$$

For $\kappa=1$ and $\rho=0$, an exact solution in $1 \mathrm{~d}$ is given by (compare [23], e.g.)

$$
\tilde{u}(s)=\ln \left(\frac{1+\cos (s)}{1-\cos (s)}\right) .
$$

For $a=(1.0,2.0) / \sqrt{5} \in \mathbb{R}^{2}$, we consider here the exact $2 \mathrm{~d}$ solution $u(x)=\tilde{u}(0.1+$ $\langle x, a\rangle)$ on the unit square $\Omega=(0,1)^{2}$. The direction $a$ was chosen such that the gradient is not aligned with any mesh side. Function $u$ attains its maximum in the origin, $u(0) \approx 6$, which implies $f(u(0))=\sinh (u(0)) \approx 200$. Figure 5.1 shows the convergence of AFEM for piecewise linear, quadratic, and cubic approximation. For each polynomial degree, the decay of estimate and exact error, together with a straight line giving the optimal decay, are depicted. For this smooth nonlinearity, AFEM is able to attain the optimal decay rate after a few iterations, when the behavior near the origin is resolved.
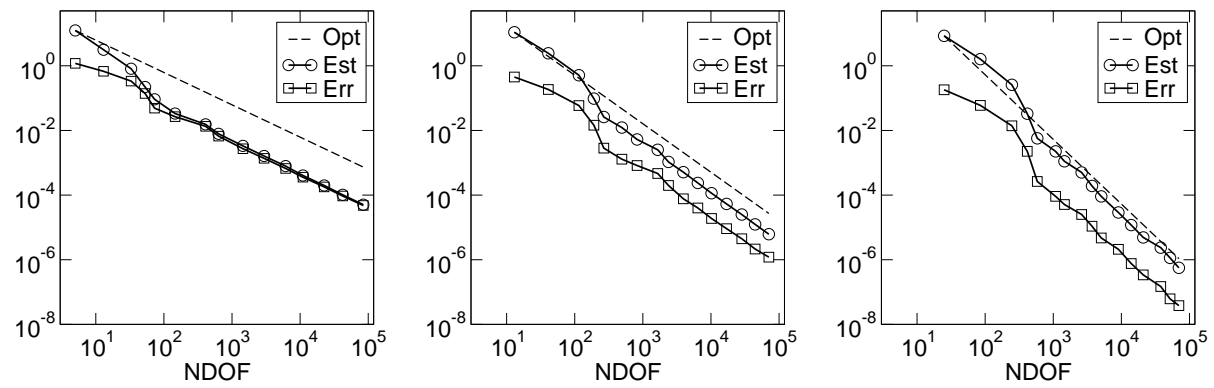

FiguRE 5.1. sinh nonlinearity: Error estimate, exact error, and optimal decay, for piecewise linear (left), quadratic (middle), and cubic elements (right). AFEM achieves optimal decay rate after a few iterations, when the behavior near the origin is resolved.

5.3. Free boundary problem. We now consider exact solutions of the form

$$
u_{R, \alpha}(x)=(|x|-R)_{+}^{\alpha},
$$

on a bounded domain, where $s_{+}:=\max \{s, 0\}$ denotes the nonnegative part of $s$. The solution attaches to the zero level on the ball of radius $R$ around 0 , the contact set; thus $\partial B(0, R) \cap \Omega$ is the free boundary. The corresponding Dirichlet problem is

$$
-\Delta u(x)+f(x, u)=0 \quad \text { in } \Omega \subset \mathbb{R}^{d}, \quad u=u_{R, \alpha} \quad \text { on } \partial \Omega
$$


with nonlinearity

$$
f(x, u)=\alpha\left(\alpha-1+(d-1) \frac{(|x|-R)_{+}}{|x|}\right) u_{+}^{\frac{\alpha-2}{\alpha}},
$$

For $\alpha>2$, the nonlinearity is Hölder $\beta$ with $\beta=\frac{\alpha-2}{\alpha}<1$ but nondifferentiable at 0 . Problems of this form arise in reaction in porous media and have been studied in $[3,22]$.

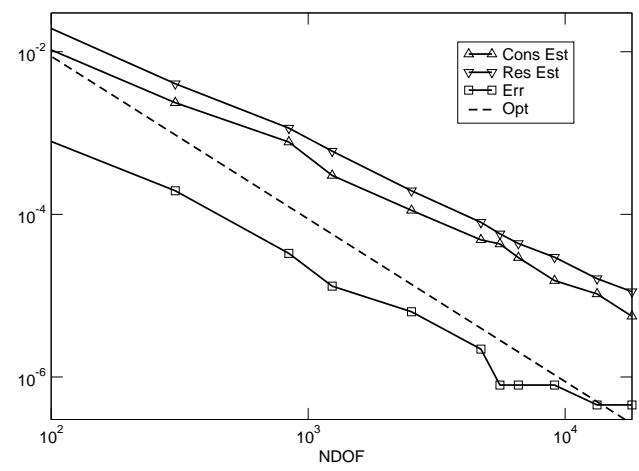

FiguRE 5.2. Free boundary problem: Convergence of consistency estimator (upper curve), residual estimator (middle curve) and error (lower curve) for AFEM, together with dashed line showing optimal decay versus number of degrees of freedom, for piecewise cubic approximation.

Experiments were performed for $d=2,3$ on $\Omega=(0,1)^{d}$ with $\alpha=2.5$ and $R=0.5$. As the exact solution is known, we can compare the error estimate with the exact error $\left\|u-u_{h}\right\|_{\infty ; \Omega}$. Figure 5.2 depicts the suboptimal decay of consistency estimator, residual estimator, and pointwise error for AFEM with $\mathbb{P}_{3}$ elements; recall that a suboptimal decay was already observed for the linear case in Remark 4.4. Figure 5.3 shows discrete solutions and meshes for two different iterations of AFEM. In the interior of the contact region, for both $d=2,3$, mesh refinement is mostly dictated by mesh conformity. Figure 5.4 illustrates this effect in $3 \mathrm{~d}$ along with isolines of solutions. Since $u \in W_{\infty}^{2}(\Omega) \backslash W_{\infty}^{3}(\Omega)$, quadratic approximation detects the lack of regularity across the free boundary and refines accordingly (see Figures 5.3 and 5.4).

\subsection{Boundary layer problem. We finally consider the problem}

$$
-\Delta u(x)-p(x) u(x)_{+}^{-\gamma}=0 \quad \text { in } \Omega \subset \mathbb{R}^{d}, \quad u=0 \quad \text { on } \partial \Omega,
$$

with $p(x) \geq 0$ and $\gamma>0$. This equation is used to model the behavior of pseudoplastic fluids $[8,14]$, and is somewhat related to the black holes equation, for which $\gamma=7$ but the boundary condition is of Robin type [4].

Due to the blow-up behavior of the negative power $u^{-\gamma}$ for $u \rightarrow 0$, the function $u \mapsto f(x, u)=-p(x) u_{+}^{-\gamma}$ is not continuous in $\mathbb{R}$ and does not fit our theory; in particular, functions $I_{h}^{q}\left(f\left(\cdot, u_{h}\right)\right)$ and $I_{h}^{q-1}\left(f\left(\cdot, u_{h}\right)\right)$ would be undefined on $\partial \Omega$ for all $\gamma$. We circumvent this matter upon considering the regularized problem

$$
-\Delta u_{\varepsilon}(x)+f_{\varepsilon}\left(x, u_{\varepsilon}(x)\right)=0 \quad \text { in } \Omega \subset \mathbb{R}^{d}, \quad u_{\varepsilon}=0 \quad \text { on } \partial \Omega,
$$



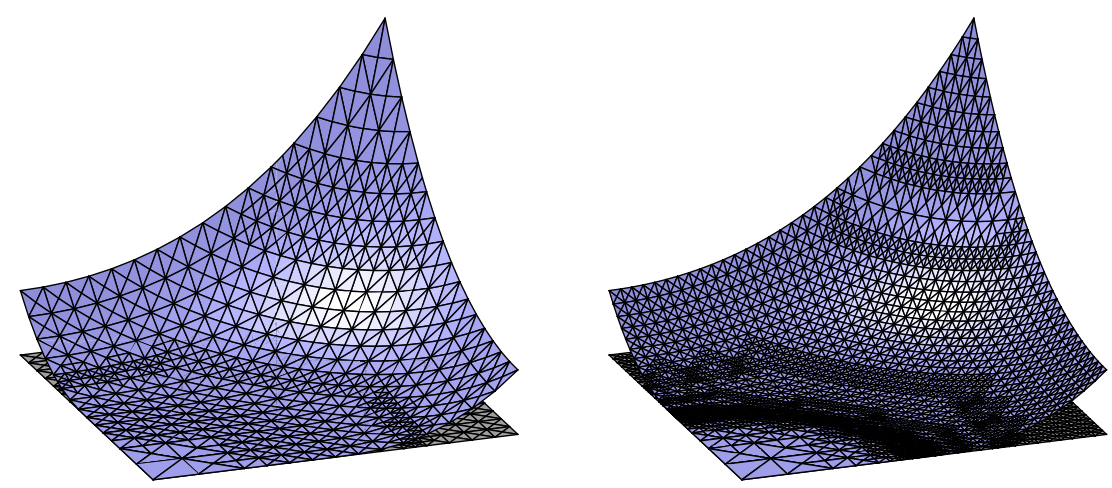

Figure 5.3. Free boundary problem in 2D: Solutions and meshes from iterations 5 and 8 of AFEM using $\ell=2$, with 2557 (11587) unknowns, error 2.82E-05 (1.64E-06). The solutions attach to zero values in the circle $B(0,0.5)$, the contact region, where the mesh remains rather coarse and is mostly driven by mesh conformity.

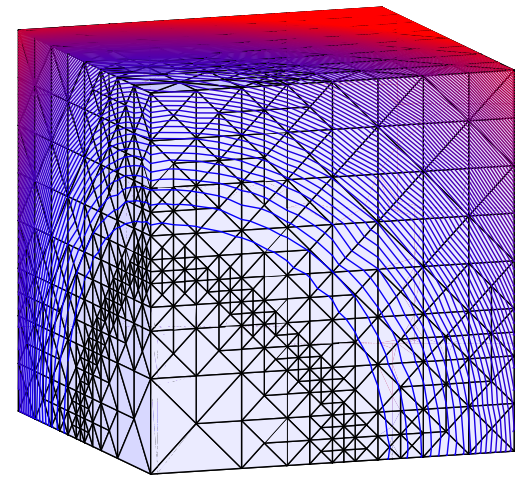

FiguRE 5.4. Free boundary problem in 3D: Mesh for piecewise quadratic elements with 35812 unknowns, and isolines of solution (at multiples of 0.01 ). The mesh remains rather coarse in the contact region $B(0,0.5)$.

where

$$
f_{\varepsilon}(x, s)=-p(x) \max (s, \varepsilon)^{-\gamma} .
$$

Since the solution $u$ is Hölder continuous for all $\gamma>0$, the question arises whether or not $u_{\varepsilon}$ converges to $u$ in $L_{\infty}(\Omega)$ with a prescribed rate. Before we explore this crucial issue, let us pause to comment on the boundary behavior of $u$ as a function of $\gamma$ for $p(x)$ uniformly positive; $u$ is smooth in the interior of $\Omega$, depending on the regularity of $p$. Lazer and McKenna [16] show the following facts:

- for $0<\gamma<1, u$ dominates $\operatorname{dist}(x, \partial \Omega)$, whereas for $\gamma>1$ it behaves exactly like $\operatorname{dist}(x, \partial \Omega)^{\frac{2}{1+\gamma}}$

- $u \in \stackrel{\circ}{H}^{1}(\Omega)$ if and only if $\gamma<3$ but $u \notin C^{1}(\bar{\Omega})$ if $\gamma>1$;

- the boundary behavior of $u$ can be compensated by $p(x)$ tending to 0 with a prescribed order. 
We examine now the rate of convergence of $u_{\varepsilon}$ to $u$. We claim that

$$
0 \leq u-u_{\varepsilon} \leq \varepsilon \quad \text { in } \Omega
$$

note that there is no constant in (5.1) and that $p$ plays no role on it. This result can be proved upon modifying a technique developed by Nochetto [18], which is briefly described here. Consider two regularized solutions $u_{\delta}$ and $u_{\varepsilon}$ for $\delta<\varepsilon$, and denote $e:=u_{\delta}-u_{\varepsilon}$. We observe that both functions $u_{\delta}$ and $u_{\varepsilon}$ are weak solutions and in fact they are globally Lipschitz. If we take the difference of the corresponding PDE and multiply by the test function $e^{2 k+1} \in \stackrel{\circ}{H}^{1}(\Omega)$ with $k$ an integer, then we obtain

$$
\int_{\Omega} \nabla e \nabla e^{2 k+1}+\left(f_{\delta}\left(\cdot, u_{\delta}\right)-f_{\varepsilon}\left(\cdot, u_{\varepsilon}\right)\right)\left(u_{\delta}-u_{\varepsilon}\right) e^{2 k}=0 .
$$

We first estimate the nonlinear term. Notice that if $u_{\delta} \geq \varepsilon$, then $f_{\delta}\left(\cdot, u_{\delta}\right)=f_{\varepsilon}\left(\cdot, u_{\delta}\right)$ and the nonlinear term is nonnegative by monotonicity. If $0 \leq u_{\delta} \leq \varepsilon$, instead, but $u_{\varepsilon} \geq \varepsilon$, then $f_{\varepsilon}\left(\cdot, u_{\varepsilon}\right)=f_{\delta}\left(\cdot, u_{\varepsilon}\right)$ and the nonlinear term is again nonnegative. The only case left is $0 \leq u_{\delta}, u_{\varepsilon} \leq \varepsilon$, for which we have the bound

$$
\int_{\left\{0 \leq u_{\delta} \leq \varepsilon\right\}} p(x)\left(\delta^{-\gamma}-\varepsilon^{-\gamma}\right)\left(u_{\delta}-u_{\varepsilon}\right)^{2 k+1} \leq C \varepsilon^{2 k+1} \delta^{-\gamma}\left|\left\{0 \leq u_{\delta} \leq \varepsilon\right\}\right| \leq C \delta^{-\gamma} \varepsilon^{2 k+1}
$$

for all $\gamma>0$. On the other hand, the gradient term can be bounded via Poincaré inequality as follows:

$$
\int_{\Omega} \nabla e \nabla e^{2 k+1}=\frac{2 k+1}{(k+1)^{2}} \int_{\Omega}\left|\nabla e^{k+1}\right|^{2} \geq C \frac{2 k+1}{(k+1)^{2}} \int_{\Omega}\left|e^{2(k+1)}\right| .
$$

Collecting the two estimates above, we arrive at

$$
\int_{\Omega}\left|e^{2(k+1)}\right| \leq C \frac{(k+1)^{2}}{2 k+1} \delta^{-\gamma} \varepsilon^{2 k+1} .
$$

Computing the power $\frac{1}{2(k+1)}$ on both sides and taking the limit as $k \rightarrow \infty$ yields $\left\|u_{\delta}-u_{\varepsilon}\right\|_{\infty ; \Omega} \leq \varepsilon$. We now take the limit as $\delta \rightarrow 0$, and use the fact that $u_{\delta} \rightarrow$ $u$ uniformly, to deduce the bound $\left\|u-u_{\varepsilon}\right\|_{\infty ; \Omega} \leq \varepsilon$. To prove the claim (5.1), it remains to show that $u \geq u_{\varepsilon}$. This follows from a weak maximum principle argument because $f_{\varepsilon}(x, s) \geq f(x, s)$ implies

$$
-\Delta u+f_{\varepsilon}(x, u) \geq-\Delta u+f(x, u)=0,
$$

whence $u$ is a supersolution of the regularized problem.

We finally consider the example of Henson and Shaker [14] with

$$
p(x)=\max \left(0.25-x_{1}, 0, x_{1}-0.75\right),
$$

on $\Omega=(0,1)^{2}$. The solution exhibits a boundary layer where $p>0$ and a smooth boundary behavior where $p=0$. For positive $\varepsilon$, both the Newton solver and AFEM work without any problems. Of course, the smaller $\varepsilon$, the stronger local refinement is generated near the boundary where $p>0$. The mesh refinement is now mostly dictated by the consistency error indicator which accounts for quadrature error. The computed solution for $\gamma=0.5$ and $\varepsilon=0.001$, together with the corresponding locally refined mesh, are shown in Figure 5.5. 

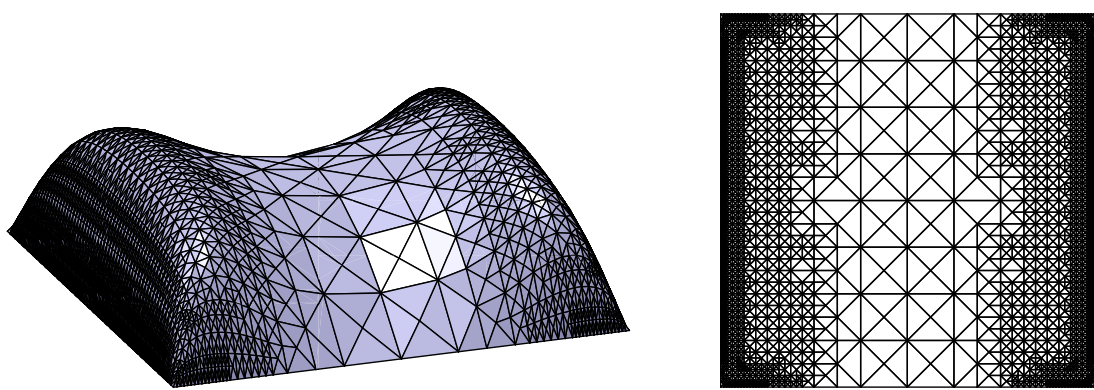

FiguRE 5.5. Boundary layer problem (scaled by factor 20) and corresponding mesh for piecewise linear approximation with 3285 unknowns and estimated error $\approx 6 e-4$. Compare the boundary layer effect for $p(x)>0$ with the smooth behavior where $p(x)=0$.

\section{ACKNOWLEDGMENTS}

The authors were partially supported in 2003 by the program "Computational Challenges in Partial Differential Equations" at the Isaac Newton Institute for Mathematical Sciences, Cambridge, UK. They were also partially supported as follows: the first author by the NSF grant DMS-0204670; the second and third authors by the DAAD grant "Efficient Finite Element Methods for Solid and Fluid Mechanics Computations"; the last author by Italian MIUR Cofin 2003 "Modellistica numerica per il calcolo scientifico e applicazioni avanzate" and Cofin 2004 "Metodi numerici avanzati per equazioni alle derivate parziali di interesse applicativo".

\section{REFERENCES}

[1] R. A. Adams, Sobolev spaces, vol. 65 of Pure and Applied Mathematics, Academic Press, Inc., a subsidiary of Harcourt Brace Jovanovich, Publishers, New York - San Francisco London, 1975.

[2] M. Ainsworth And J. T. Oden, A posteriori error estimation in finite element analysis, John Wiley \& Sons, Inc., 2000.

[3] H. W. Alt And D. Phillips, A free boundary problem for semilinear elliptic equations, J. Reine Angew. Math. 368, (1986), pp. 63-107.

[4] D. N. Arnold, A. Mukherjee, and L. Pouly, Adaptive finite elements and colliding black holes, Numerical analysis 1997 (Dundee), 1-15, Pitman Res. Notes Math. Ser., 380, Longman, Harlow, 1998.

[5] I. BABUŠKA AND W. RheinBoldt, Error estimates for adaptive finite element computations, SIAM J. Numer. Anal., 15 (1978), pp. 736-754.

[6] S. C. BRenner And L. R. ScotT, The mathematical theory of finite element methods, Springer (2002).

[7] H. Brezis and W. Strauss, Semilinear second-order elliptic equations in $L^{1}$, J. Math. Soc. Japan, 25 (1973), pp. 565-590.

[8] A. J. Callegari and A. Nachman, A nonlinear singular boundary value problem in the theory of pseudoplastic fluids, SIAM J. Appl. Math. 30, (1980), pp. 275-281.

[9] P. G. Ciarlet The finite element method for elliptic problems, North-Holland (1980).

[10] E. DARI, R. G. DuRÁn, AND C. PADRA, Maximum norm error estimators for threedimensional elliptic problems, SIAM J. Numer. Anal., 37 (2000), pp. 683-700.

[11] L. C. Evans, Partial differential equations, Graduate Studies in Mathematics 19, AMS (1998).

[12] D. Gilbarg And N. S. Trudinger, Elliptic partial diffferential equations of second order, Springer (1983).

[13] P. GRISvard, Elliptic problems in nonsmooth domains, Pitman (1985). 
[14] V. E. Henson And A. W. Shaker, Theory and numerics for a semilinear PDE in the theory of pseudoplastic fluids, Appl. Anal. 63, (1996), pp. 271-285.

[15] D. Kinderlehrer and G. Stampacchia, An introduction to variational inequalities and their applications, vol. 88 of Pure Appl. Math., Academic Press, New York, 1980.

[16] A. C. LAzer AND P. J. MCKenna, On a singular nonlinear elliptic boundary value problem, Proc. AMS 111 (1991), pp. 721-730.

[17] R. H. Nochetto, Pointwise a posteriori error estimates for elliptic problems on highly graded meshes, Math. Comp., 64 (1995), pp. 1-22.

[18] R. H. Nochetтo, Sharp $L^{\infty}$-error estimates for semilinear elliptic problems with free boundaries, Numer. Math., 54 (1988), pp. 243-255.

[19] R. H. Nochetto, K. G. Siebert, and A. Veeser, Pointwise a posteriori error control for elliptic obstacle problems, Numer. Math., 95 (2003), pp. 163-195.

[20] R. H. Nochetto, K. G. Siebert, And A. Veeser, Fully localized a posteriori error estimators and barrier sets for contact problems, SIAM J. Numer. Anal., 42 (2005), pp. 2118-2135.

[21] J. M. Ortega And W. C. Rheinboldt, Iterative solution of nonlinear equations in several variables, Academic Press, 1970.

[22] D. PHILlips, Hausdorff measure estimates of a free boundary for a minimum problem, Comm. Partial Differential Equations, 8 (1983), pp. 1409-1454.

[23] W. B. Richardson JR., Sobolev preconditioning for the Poisson-Boltzmann equation, Comput. Meth. Appl. Mech. Engrg. 181 (2000), pp. 425-436.

[24] A. Schmidt And K. G. Siebert, Design of adaptive finite element software: The finite element toolbox ALBERTA, Springer LNCSE Series 42, 2005.

[25] A. Schmidt and K. G. Siebert, ALBERT - Software for scientific computations and applications, Acta Math. Univ. Comenianae 70 (2001), pp. 105-122.

[26] R. Verfürth, A review of a posteriori error estimation and adaptive mesh-refinement techniques, Wiley \& Sons, Teubner, 1996.

Ricardo H. Nochetto, Department of Mathematics and Institute of Physical Science and Technology, University of Maryland, College Park, MD 20742, USA.

$U R L:$ http://www.math.umd.edu/ rhn

E-mail address: rhn@math.umd.edu

Alfred Schmidt, Zentrum für Technomathematik Fachbereich 3 Mathematik und Informatik, Universität Bremen, Postfach 3304 40, D-28334 Bremen, Germany.

URL: http://www.math.uni-bremen.de/ ${ }^{\sim}$ schmidt

E-mail address: Schmidt@math.uni-bremen.de

Kunibert G. Siebert, Institut für Mathematik, Universität Augsburg, UniversitätsStrasse 14, D-86159 Augsburg, Germany.

URL: http://scicomp.math.uni-augsburg.de/Siebert/

E-mail address: siebert@math.uni-augsburg.de

Andreas Veeser, Dipartimento di Matematica, Università degli Studi di Milano, Via C. Saldini 50, 20133 Milano, Italy.

E-mail address: veeser@mat.unimi.it 